\title{
Level truncation analysis of a simple tachyon vacuum solution in cubic superstring field theory
}

\section{E. Aldo Arroyo}

Centro de Ciências Naturais e Humanas, Universidade Federal do ABC,

Santo André, 09210-170 São Paulo, SP, Brazil

E-mail: aldo.arroyo@ufabc.edu.br

ABSTRACT: We evaluate the vacuum energy of a simple tachyon vacuum solution using the level truncation scheme in cubic superstring field theory. By truncating the standard Virasoro $L_{0}$ level expansion of the solution, we obtain a value of the vacuum energy in agreement with Sen's first conjecture.

Keywords: Tachyon Condensation, String Field Theory

ARXIV EPRINT: 1409.1890 


\section{Contents}

1 Introduction

2 A simple analytic solution for tachyon condensation in cubic superstring field theory

2.1 Computation of the kinetic term 4

2.2 Computation of the cubic term 5

2.3 Computation of Ellwood's gauge invariant 6

$\begin{array}{lll}3 & \text { Level expansion analysis } & \mathbf{7}\end{array}$

$\begin{array}{lll}3.1 & L_{0} \text { level expansion of the simple tachyon vacuum solution } & 7\end{array}$

$\begin{array}{ll}3.2 & \text { Level truncation evaluation of the vacuum energy }\end{array}$

4 Summary and discussion $\quad 11$

A Details on the level truncation evaluation of the vacuum energy 13

\section{Introduction}

The analytic solution for tachyon condensation [1] in Witten's open bosonic string field theory [2] has provided analytical and numerical tools to analyze several classical solutions of the string field equations of motion [3]-[24]. In the framework of the modified cubic superstring field theory [25], the analytic construction of the tachyon vacuum solution was analyzed by Erler [26]. Using the $K B c \gamma$ subalgebra introduced in [27, 28], many gauge equivalent tachyon vacuum solutions [29-33] have been proposed and the computation of the energy associated to these solutions was performed giving results in agreement with Sen's conjecture. The analysis of multibrane solutions has been given in a set of two papers [34, 35]. In the case of Berkovits non-polynomial open superstring field theory [36], the analytic construction of the tachyon vacuum solution, based on an extension of the $K B c \gamma$ subalgebra, has been studied by Erler [37].

In the bosonic context, the well known solutions, i.e., Schnabl's [1] and the simple ErlerSchnabl's analytic solutions for tachyon condensation [4] were used to analytically test Sen's conjecture. Apart from these analytical computations, further numerical evidence has been provided by the so-called level truncation analysis [4, 24]. In the superstring case, only analytical calculations have been performed with the solutions [26, 30,37] and the results were in agreement with Sen's conjecture. So that the tachyon vacuum solution from an analytic perspective appears to be as regular as Schnabl's original solution for the bosonic string. Nevertheless, from the perspective of the level expansion the situation is unknown, 
because the analysis of the energy for the tachyon vacuum solution in cubic superstring field theory using the usual $L_{0}$ level expansion has not yet been carried out.

In this paper, we will analyze a simple analytic solution for tachyon condensation in cubic superstring field theory. This solution is written in terms of the elements of the $K B c \gamma$ subalgebra

$$
\Psi_{\lambda}=\frac{1}{\sqrt{1+\lambda K}}\left[\frac{1}{\lambda} c+c K B c+B \gamma^{2}\right] \frac{1}{\sqrt{1+\lambda K}}
$$

The solution (1.1) with $\lambda=1$ has been studied by Gorbachev [30], and his main results were: (i) the analytical computation of the energy leads to a value in agreement with Sen's first conjecture, and (ii) the proof of the absence of physical excitations in the vicinity of the tachyon vacuum. Let us point out that to obtain the result (i), the validity of the equation of motion contracted with the solution itself was assumed. However, to explicitly test the validity of this assumption, the cubic term of the action must be computed. By employing the solution (1.1) with a generic $\lambda$, we will compute the kinetic $\left\langle\Psi_{\lambda} Q \Psi_{\lambda}\right\rangle$ and the cubic terms $\left\langle\Psi_{\lambda} \Psi_{\lambda} \Psi_{\lambda}\right\rangle$, and test the validity of the equation of motion contracted with the solution itself, namely $\left\langle\Psi_{\lambda} Q \Psi_{\lambda}\right\rangle+\left\langle\Psi_{\lambda} \Psi_{\lambda} \Psi_{\lambda}\right\rangle=0$. Additionally, we will evaluate Ellwood's gauge invariant observable.

Apart from the evaluation of the cubic term and Ellwood's gauge invariant observable, we will study the solution using the traditional Virasoro $L_{0}$ level truncation scheme [3842]. This analysis is important since we want to know if the solution behaves as a regular element in the state space constructed out of Fock states. Specifically the analysis of the coefficients appearing in the $L_{0}$ level expansion provides one criterion for the solution being well defined [5, 24]. Furthermore the $L_{0}$ level expansion of the solution will provide us with an additional way to numerically test Sen's first conjecture [24, 43-45].

The main motivation for developing a level truncation analysis of a simple tachyon vacuum solution in cubic superstring field theory is to prepare a numerical background in order to analyze more cumbersome solutions, such as the multibrane solutions [34, 35], and the recently proposed Erler's analytic solution for tachyon condensation in Berkovits nonpolynomial open superstring field theory [37]. Since the algebraic structure of Berkovits theory is similar to the cubic superstring field theory, the results of our work can be naturally extended, however the presence of a non-polynomial action in Berkovits theory, will bring us challenges in the level truncation analysis of Erler's solution.

This paper is organized as follows. In section 2, we review the construction of the simple tachyon vacuum solution in cubic superstring field theory and compute the kinetic and the cubic terms of the action. Ellwood's gauge invariant observable is also evaluated. In section 3, we present the level expansion analysis of the solution. By truncating the standard Virasoro $L_{0}$ level expansion of the solution, we compute the vacuum energy and obtain a result in agreement with Sen's first conjecture. In section 4, a summary and further directions of exploration are given. Some details regarding the level truncation evaluation of the vacuum energy are given in the appendix A. 


\section{A simple analytic solution for tachyon condensation in cubic super- string field theory}

In this section, we are going to review the construction of the simple tachyon vacuum solution in cubic superstring field theory [27]. Let us start by writing a string field $\Psi$ as a pure gauge form

$$
\Psi=\mathcal{U Q U}^{-1}
$$

so that $\Psi$ formally satisfies the string field equation of motion $Q \Psi+\Psi \Psi=0$, where $Q$ is the BRST operator of the open Neveu-Schwarz superstring theory.

We can construct a string field $\mathcal{U}$ by employing the elements of the $K B c \gamma$ subalgebra. The basic string fields of this subalgebra are given by [26-28]

$$
\begin{aligned}
K & =\frac{1}{2} \hat{\mathcal{L}} U_{1}^{\dagger} U_{1}|0\rangle, \\
B & =\frac{1}{2} \hat{\mathcal{B}} U_{1}^{\dagger} U_{1}|0\rangle, \\
c & =U_{1}^{\dagger} U_{1} \tilde{c}(0)|0\rangle, \\
\gamma & =U_{1}^{\dagger} U_{1} \tilde{\gamma}(0)|0\rangle,
\end{aligned}
$$

where the operator $U_{1}^{\dagger} U_{1}$ is defined in general as $U_{r}^{\dagger} U_{r}=e^{\frac{2-r}{2}} \hat{\mathcal{L}}$. The operators $\hat{\mathcal{L}}, \hat{\mathcal{B}}, \tilde{c}(0)$ and $\tilde{\gamma}(0)$ are defined in the sliver frame, ${ }^{1}$ and they are related to the worldsheet energymomentum tensor, the $b, c$ and $\gamma$ ghosts fields respectively. For instance, the operators $\hat{\mathcal{L}}$ and $\hat{\mathcal{B}}$ are given by

$$
\begin{aligned}
& \hat{\mathcal{L}} \equiv \mathcal{L}_{0}+\mathcal{L}_{0}^{\dagger}=\oint \frac{d z}{2 \pi i}\left(1+z^{2}\right)(\arctan z+\operatorname{arccot} z) T(z) \\
& \hat{\mathcal{B}} \equiv \mathcal{B}_{0}+\mathcal{B}_{0}^{\dagger}=\oint \frac{d z}{2 \pi i}\left(1+z^{2}\right)(\arctan z+\operatorname{arccot} z) b(z)
\end{aligned}
$$

Using these definitions, we can show that the basic elements of the $K B c \gamma$ subalgebra (2.2)-(2.5) satisfy the algebraic relations

$$
\begin{aligned}
& \{B, c\}=1, \quad[B, K]=0, \quad B^{2}=c^{2}=0, \\
& \partial c=[K, c], \quad \partial \gamma=[K, \gamma], \quad[c, \gamma]=0, \quad[B, \gamma]=0,
\end{aligned}
$$

and have the following BRST variations

$$
Q K=0, \quad Q B=K, \quad Q c=c K c-\gamma^{2}, \quad Q \gamma=c \partial \gamma-\frac{1}{2} \gamma \partial c .
$$

Employing the elements of the $K B c \gamma$ subalgebra, let us write the string field $\mathcal{U}$ as follows

$$
\mathcal{U}=1-F B c F \quad, \quad \mathcal{U}^{-1}=1+\frac{F}{1-F^{2}} B c F,
$$

\footnotetext{
${ }^{1}$ Remember that a point in the upper half plane $z$ is mapped to a point in the sliver frame $\tilde{z}$ via the conformal mapping $\tilde{z}=\frac{2}{\pi} \arctan z$. Note that we are using the convention of [4] for the conformal mapping.
} 
where $F$ is a function of $K$ given by

$$
F(K)=\frac{1}{\sqrt{1+\lambda K}}
$$

Using this string field $\mathcal{U}$ and the relations (2.8)-(2.10), we can derive the following solution

$$
\Psi_{\lambda}=\mathcal{U Q U}^{-1}=\frac{1}{\sqrt{1+\lambda K}}\left[\frac{1}{\lambda} c+c K B c+B \gamma^{2}\right] \frac{1}{\sqrt{1+\lambda K}} .
$$

The solution (2.13) with $\lambda=1$ has been analyzed by Gorbachev [30], and his main results were: (i) the analytical computation of the energy leads to a value in agreement with Sen's first conjecture, and (ii) the proof of the absence of physical excitations in the vicinity of the tachyon vacuum. Let us point out that to obtain the result (i), the validity of the equation of motion contracted with the solution itself was assumed. To explicitly test the validity of this assumption, the cubic term of the action must be computed. Apart from the evaluation of the cubic term, it remains to analyze the solution using the traditional Virasoro $L_{0}$ level truncation scheme. This analysis is important since we want to know if the solution behaves as a regular element in the state space constructed out of Fock states.

In the next subsections, by employing the solution (2.13) with a generic $\lambda$, we are going to compute the kinetic term $\left\langle\Psi_{\lambda} Q \Psi_{\lambda}\right\rangle$ and the cubic term $\left\langle\Psi_{\lambda} \Psi_{\lambda} \Psi_{\lambda}\right\rangle$, and we will test the validity of the equation of motion contracted with the solution itself, namely $\left\langle\Psi_{\lambda} Q \Psi_{\lambda}\right\rangle+\left\langle\Psi_{\lambda} \Psi_{\lambda} \Psi_{\lambda}\right\rangle=0$. Additionally, we will evaluate Ellwood's gauge invariant observable. However, the main result of our paper will be presented in section 3, i.e., the level truncation analysis of the solution.

\subsection{Computation of the kinetic term}

In this subsection, we are going to compute the kinetic term of the action. Since we have that $Q[B c]=c K B c+B \gamma^{2}$, to simplify the computation it will be useful to write the solution (2.13) in the following way

$$
\Psi_{\lambda}=\frac{1}{\sqrt{1+\lambda K}} \frac{c}{\lambda} \frac{1}{\sqrt{1+\lambda K}}+Q\left\{\frac{1}{\sqrt{1+\lambda K}} B c \frac{1}{\sqrt{1+\lambda K}}\right\} .
$$

Plugging this solution into the definition of the kinetic term $\left\langle\Psi_{\lambda} Q \Psi_{\lambda}\right\rangle$, we get

$$
\left\langle\Psi_{\lambda} Q \Psi_{\lambda}\right\rangle=\frac{1}{\lambda^{2}}\left\langle c \frac{1}{1+\lambda K} Q c \frac{1}{1+\lambda K}\right\rangle .
$$

The action of the BRST operator on the $c$ field is $Q c=c K c-\gamma^{2}$. Since the nonvanishing correlators for elements in the $K B c \gamma$ subalgebra, in cubic superstring field theory, are proportional to $\left\langle c F_{1}(K) \gamma^{2} F_{2}(K)\right\rangle$ or $\left\langle B \gamma^{2} F_{1}(K) c F_{2}(K) c F_{3}(K)\right\rangle$, the non-vanishing contribution to the kinetic term $(2.15)$ is given by

$$
\left\langle\Psi_{\lambda} Q \Psi_{\lambda}\right\rangle=-\frac{1}{\lambda^{2}}\left\langle c \frac{1}{1+\lambda K} \gamma^{2} \frac{1}{1+\lambda K}\right\rangle .
$$

Let us write the integral representation of the function $1 /(1+\lambda K)$,

$$
\frac{1}{1+\lambda K}=\int_{0}^{\infty} d t e^{-t(1+\lambda K)}=\int_{0}^{\infty} d t e^{-t} \Omega^{\lambda t}
$$


where $\Omega=e^{-K}$. Plugging this integral representation into the equation (2.16), we obtain

$$
\left\langle\Psi_{\lambda} Q \Psi_{\lambda}\right\rangle=-\frac{1}{\lambda^{2}} \int_{0}^{\infty} d t_{1} d t_{2} e^{-t_{1}-t_{2}}\left\langle c \Omega^{\lambda t_{1}} \gamma^{2} \Omega^{\lambda t_{2}}\right\rangle
$$

where the correlator $\left\langle c \Omega^{\lambda t_{1}} \gamma^{2} \Omega^{\lambda t_{2}}\right\rangle$ is given by $[26,27]$

$$
\left\langle c \Omega^{\lambda t_{1}} \gamma^{2} \Omega^{\lambda t_{2}}\right\rangle=\frac{\lambda^{2}\left(t_{1}+t_{2}\right)^{2}}{2 \pi^{2}},
$$

so that plugging equation (2.19) into equation (2.18), we get

$$
\left\langle\Psi_{\lambda} Q \Psi_{\lambda}\right\rangle=-\frac{1}{2 \pi^{2}} \int_{0}^{\infty} d t_{1} d t_{2} e^{-t_{1}-t_{2}}\left(t_{1}+t_{2}\right)^{2},
$$

where the integral has the value

$$
\int_{0}^{\infty} d t_{1} d t_{2} e^{-t_{1}-t_{2}}\left(t_{1}+t_{2}\right)^{2}=6
$$

therefore the value of the kinetic term is

$$
\left\langle\Psi_{\lambda} Q \Psi_{\lambda}\right\rangle=-\frac{3}{\pi^{2}}
$$

as expected.

\subsection{Computation of the cubic term}

In this subsection, we are going to compute the cubic term of the action. Plugging the solution (2.14) into the definition of the cubic term $\left\langle\Psi_{\lambda} \Psi_{\lambda} \Psi_{\lambda}\right\rangle$, and using the fact that the only non-vanishing correlators are proportional to $\left\langle c F_{1}(K) \gamma^{2} F_{2}(K)\right\rangle$ or $\left\langle B \gamma^{2} F_{1}(K) c F_{2}(K) c F_{3}(K)\right\rangle$, after performing some algebraic manipulations, we obtain

$$
\begin{aligned}
\left\langle\Psi_{\lambda} \Psi_{\lambda} \Psi_{\lambda}\right\rangle= & \frac{3}{\lambda^{2}}\left\langle B \gamma^{2} \frac{1}{1+\lambda K} c \frac{1}{1+\lambda K} c \frac{1}{1+\lambda K}\right\rangle+\frac{3}{\lambda}\left\langle B \gamma^{2} \frac{1}{1+\lambda K} c \frac{1}{1+\lambda K} c \frac{K}{1+\lambda K}\right\rangle \\
& +\frac{3}{\lambda}\left\langle B \gamma^{2} \frac{K}{1+\lambda K} c \frac{1}{1+\lambda K} c \frac{1}{1+\lambda K}\right\rangle .
\end{aligned}
$$

Let us write the integral representation for the corresponding functions,

$$
\begin{aligned}
& \frac{1}{1+\lambda K}=\int_{0}^{\infty} d t e^{-t(1+\lambda K)}=\int_{0}^{\infty} d t e^{-t} \Omega^{\lambda t} \\
& \frac{K}{1+\lambda K}=-\frac{1}{\lambda} \int_{0}^{\infty} d t e^{-t} \frac{\partial}{\partial t}\left(e^{-t \lambda K}\right)=-\frac{1}{\lambda} \int_{0}^{\infty} d t e^{-t} \frac{\partial}{\partial t} \Omega^{\lambda t} .
\end{aligned}
$$

Plugging these integral representations into the equation (2.23), we get

$$
\left\langle\Psi_{\lambda} \Psi_{\lambda} \Psi_{\lambda}\right\rangle=\frac{3}{\lambda^{2}} \int_{0}^{\infty} d t_{1} d t_{2} d t_{3} e^{-t_{1}-t_{2}-t_{3}}\left(1-\frac{\partial}{\partial t_{1}}-\frac{\partial}{\partial t_{3}}\right)\left\langle B \gamma^{2} \Omega^{\lambda t_{1}} c \Omega^{\lambda t_{2}} c \Omega^{\lambda t_{3}}\right\rangle,
$$

where the correlator $\left\langle B \gamma^{2} \Omega^{\lambda t_{1}} c \Omega^{\lambda t_{2}} c \Omega^{\lambda t_{3}}\right\rangle$ is given by [26, 27]

$$
\left\langle B \gamma^{2} \Omega^{\lambda t_{1}} c \Omega^{\lambda t_{2}} c \Omega^{\lambda t_{3}}\right\rangle=\frac{\lambda^{2}\left(t_{1}+t_{2}+t_{3}\right) t_{2}}{2 \pi^{2}},
$$


so that plugging equation (2.27) into equation (2.26), we get

$$
\left\langle\Psi_{\lambda} \Psi_{\lambda} \Psi_{\lambda}\right\rangle=\frac{3}{2 \pi^{2}} \int_{0}^{\infty} d t_{1} d t_{2} d t_{3} e^{-t_{1}-t_{2}-t_{3}}\left(t_{1}+t_{2}+t_{3}-2\right) t_{2},
$$

where the integral has the value

$$
\int_{0}^{\infty} d t_{1} d t_{2} d t_{3} e^{-t_{1}-t_{2}-t_{3}}\left(t_{1}+t_{2}+t_{3}-2\right) t_{2}=2,
$$

therefore the value of the cubic term is

$$
\left\langle\Psi_{\lambda} \Psi_{\lambda} \Psi_{\lambda}\right\rangle=+\frac{3}{\pi^{2}},
$$

as expected.

Employing the result of the kinetic term (2.22) and the cubic term (2.30), we just have proven the validity of the equation of motion contracted with the solutions itself, namely: $\left\langle\Psi_{\lambda} Q \Psi_{\lambda}\right\rangle+\left\langle\Psi_{\lambda} \Psi_{\lambda} \Psi_{\lambda}\right\rangle=0$. In the next subsection, we are going to evaluate another important gauge invariant quantity.

\subsection{Computation of Ellwood's gauge invariant}

Let us evaluate Ellwood's gauge invariant overlap for the tachyon vacuum solution. For a generic solution $\Psi$, Ellwood's gauge invariant overlap is defined as

$$
W(\Psi, \mathcal{V})=\operatorname{Tr}(\Psi)
$$

where the notation $\operatorname{Tr}(\cdots)$ refers to a correlator with an on shell closed string vertex operator $\mathcal{V}(i)$ inserted at the midpoint, $\operatorname{Tr}(\Psi)=\langle\mathcal{V}(i) \Psi\rangle$. We assume the same $\mathcal{V}$ used in reference [28], this field is an NS-NS closed string vertex operator of the form

$$
\mathcal{V}(z)=c \tilde{c} e^{-\phi} e^{-\tilde{\phi}} \mathcal{O}^{\mathrm{m}},
$$

where $\mathcal{O}^{\mathrm{m}}$ is a weight $\left(\frac{1}{2}, \frac{1}{2}\right)$ superconformal matter primary field. As argued by Ellwood [17], the gauge invariant overlap represents the shift in the closed string tadpole of the solution relative to the perturbative vacuum.

Inserting the solution (2.14) into the definition of the gauge invariant overlap (2.31), the BRST exact term does not contribute, therefore we get

$$
W\left(\Psi_{\lambda}, \mathcal{V}\right)=\frac{1}{\lambda} \operatorname{Tr}\left(\frac{1}{\sqrt{1+\lambda K}} c \frac{1}{\sqrt{1+\lambda K}}\right) .
$$

Let us write the integral representation of the function $1 / \sqrt{1+\lambda K}$,

$$
\frac{1}{\sqrt{1+\lambda K}}=\frac{1}{\sqrt{\pi}} \int_{0}^{\infty} d t \frac{1}{\sqrt{t}} e^{-t(1+\lambda K)}=\frac{1}{\sqrt{\pi}} \int_{0}^{\infty} d t \frac{1}{\sqrt{t}} e^{-t} \Omega^{\lambda t} .
$$

Plugging this integral representation into the equation (2.33), we obtain

$$
W\left(\Psi_{\lambda}, \mathcal{V}\right)=\frac{1}{\lambda \pi} \int_{0}^{\infty} d t_{1} d t_{2} \frac{1}{\sqrt{t_{1} t_{2}}} e^{-t_{1}-t_{2}} \operatorname{Tr}\left(\Omega^{\lambda t_{1}} c \Omega^{\lambda t_{2}}\right) .
$$


The correlator $\operatorname{Tr}\left(\Omega^{\lambda t_{1}} c \Omega^{\lambda t_{2}}\right)$ is given by $[28,34]$

$$
\operatorname{Tr}\left(\Omega^{\lambda t_{1}} c \Omega^{\lambda t_{2}}\right)=\lambda\left(t_{1}+t_{2}\right) \operatorname{Tr}(c \Omega)
$$

where $\Omega=e^{-K}$ and $\operatorname{Tr}(c \Omega)=\langle\mathcal{V}(i \infty) c(0)\rangle_{C_{1}}$ is the expected result of the closed string tadpole on the disk. Plugging the correlator (2.36) into equation (2.35), we get

$$
W\left(\Psi_{\lambda}, \mathcal{V}\right)=\frac{1}{\pi} \int_{0}^{\infty} d t_{1} d t_{2} \frac{1}{\sqrt{t_{1} t_{2}}} e^{-t_{1}-t_{2}}\left(t_{1}+t_{2}\right) \operatorname{Tr}(c \Omega),
$$

where the integral has the value

$$
\int_{0}^{\infty} d t_{1} d t_{2} \frac{1}{\sqrt{t_{1} t_{2}}} e^{-t_{1}-t_{2}}\left(t_{1}+t_{2}\right)=\pi
$$

therefore the value of Ellwood's gauge invariant is

$$
W\left(\Psi_{\lambda}, \mathcal{V}\right)=(+1) \operatorname{Tr}(c \Omega),
$$

as expected.

Since the analytic computation of the gauge invariant quantities performed in the previous subsections leads to the desired results, the tachyon vacuum solution (2.13) from an analytic perspective appears to be as regular as Schnabl's original solution for the bosonic string. Let us see what happens from a numerical perspective. In what follows, we are going to analyze the solution using the usual $L_{0}$ level expansion scheme.

\section{Level expansion analysis}

In this section, we are going to analyze the level expansion of the simple tachyon vacuum solution (2.14). The analysis of a string field using the traditional $L_{0}$ level expansion scheme is important since this information tells us if the solution behaves as a regular element in the state space constructed out of Fock states. Specifically the analysis of the coefficients appearing in the $L_{0}$ level expansion provides one criterion for the solution being well defined $[5,24]$. Moreover the $L_{0}$ level expansion of the solution brings an additional way to numerically test Sen's first conjecture.

\section{1 $L_{0}$ level expansion of the simple tachyon vacuum solution}

To expand the simple tachyon vacuum solution (2.14) in the Virasoro basis of $L_{0}$ eigenstates, we start by writing the function $1 / \sqrt{1+\lambda K}$ as its integral representation (2.34). Plugging this integral representation (2.34) into the expression for the simple tachyon vacuum solution (2.14), we obtain

$$
\Psi_{\lambda}=\frac{1}{\pi \lambda} \int_{0}^{\infty} d s d t \frac{1}{\sqrt{s t}} e^{-s-t} \Omega^{\lambda t} c \Omega^{\lambda s}+Q\left\{\frac{1}{\pi} \int_{0}^{\infty} d s d t \frac{1}{\sqrt{s t}} e^{-s-t} \Omega^{\lambda t} B c \Omega^{\lambda s}\right\}
$$

where the wedge state $\Omega^{t}$ can be expressed in terms of the scaling operator $U_{r}[4,18]$

$$
\Omega^{t}=e^{-t K}=U_{t+1}^{\dagger} U_{t+1}|0\rangle, \quad \text { where } \quad U_{r} \equiv\left(\frac{2}{r}\right)^{\mathcal{L}_{0}} .
$$


Let us write rather general formulas which will be very useful for the $L_{0}$ level expansion analysis of a string field constructed out of elements in the $K B c \gamma$ subalgebra,

$$
\begin{aligned}
& e^{-t_{1} K} c e^{-t_{2} K} B c e^{-t_{3} K}=\frac{r \cos ^{2}\left(\frac{\pi x}{r}\right)\left(\pi(r-2 y)-r \sin \left(\frac{2 \pi y}{r}\right)\right)}{4 \pi^{2}} \widetilde{U}_{r} c\left(\frac{2 \tan \left(\frac{\pi x}{r}\right)}{r}\right)|0\rangle \\
& +\frac{r \cos ^{2}\left(\frac{\pi y}{r}\right)\left(\pi(r+2 x)+r \sin \left(\frac{2 \pi x}{r}\right)\right)}{4 \pi^{2}} \widetilde{U}_{r} c\left(\frac{2 \tan \left(\frac{\pi y}{r}\right)}{r}\right)|0\rangle \\
& +\sum_{k=1}^{\infty} \frac{(-1)^{k+1} 2^{2 k-1}\left(\frac{1}{r}\right)^{2 k-3} \cos ^{2}\left(\frac{\pi x}{r}\right) \cos ^{2}\left(\frac{\pi y}{r}\right)}{\left(4 k^{2}-1\right) \pi^{2}} \widetilde{U}_{r} b_{-2 k} c\left(\frac{2 \tan \left(\frac{\pi x}{r}\right)}{r}\right) c\left(\frac{2 \tan \left(\frac{\pi y}{r}\right)}{r}\right)|0\rangle, \\
& r=t_{1}+t_{2}+t_{3}+1, \quad x=\frac{1}{2}\left(t_{3}-t_{1}-t_{2}\right), \quad y=\frac{1}{2}\left(t_{2}+t_{3}-t_{1}\right), \\
& e^{-t K} B \gamma^{2} e^{-t K}=\frac{2}{\pi} \sum_{k=1}^{\infty} \widetilde{U}_{2 t+1} \frac{(-1)^{k+1}}{4 k^{2}-1}\left(\frac{2}{2 t+1}\right)^{2 k-1} b_{-2 k} \gamma^{2}(0)|0\rangle,
\end{aligned}
$$

where the operator $\widetilde{U}_{r}$ is defined as

$$
\widetilde{U}_{r} \equiv \cdots e^{u_{10, r} L_{-10}} e^{u_{8, r} L_{-8}} e^{u_{6, r} L_{-6}} e^{u_{4, r} L_{-4}} e^{u_{2, r} L_{-2}} .
$$

To find the coefficients $u_{n, r}$ appearing in the exponentials, we use

$$
\begin{aligned}
\frac{r}{2} \tan \left(\frac{2}{r} \arctan z\right) & =\lim _{N \rightarrow \infty}\left[f_{2, u_{2, r}} \circ f_{4, u_{4, r}} \circ f_{6, u_{6, r}} \circ f_{8, u_{8, r}} \circ f_{10, u_{10, r}} \circ \cdots \circ f_{N, u_{N, r}}(z)\right] \\
& =\lim _{N \rightarrow \infty}\left[f_{2, u_{2, r}}\left(f_{4, u_{4, r}}\left(f_{6, u_{6, r}}\left(f_{8, u_{8, r}}\left(f_{10, u_{10, r}}\left(\cdots\left(f_{N, u_{N, r}}(z)\right) \ldots\right)\right)\right)\right)\right]\right.
\end{aligned}
$$

where the function $f_{n, u_{n, r}}(z)$ is given by

$$
f_{n, u_{n, r}}(z)=\frac{z}{\left(1-u_{n, r} n z^{n}\right)^{1 / n}}
$$

Employing the set of equations (3.2)-(3.6) for the solution (3.1), we obtain

$$
\Psi_{\lambda}=\frac{1}{2 \pi^{2} \lambda} \int_{0}^{\infty} d s d t \frac{1}{\sqrt{s t}} e^{-s-t} r^{2} \cos ^{2}\left(\frac{\pi x}{r}\right) \widetilde{U}_{r} c\left(\frac{2 \tan \left(\frac{\pi x}{r}\right)}{r}\right)|0\rangle+Q_{\text {exact term }}
$$

where

$$
r=\lambda(s+t)+1, \quad x=\frac{\lambda}{2}(s-t) .
$$

Since in the evaluation of the vacuum energy, the $Q_{\text {exact term }}$ will not contribute, we only need to consider the first term on the right hand side of equation (3.9). Let us study in some detail this first term

$$
\Psi_{\lambda}^{(1)} \equiv \frac{1}{2 \pi^{2} \lambda} \int_{0}^{\infty} d s d t \frac{1}{\sqrt{s t}} e^{-s-t} r^{2} \cos ^{2}\left(\frac{\pi x}{r}\right) \widetilde{U}_{r} c\left(\frac{2 \tan \left(\frac{\pi x}{r}\right)}{r}\right)|0\rangle,
$$

with $r$ and $x$ given by equation (3.10). 
By writing the $c$ ghost in terms of its modes $c(z)=\sum_{m} c_{m} / z^{m-1}$ and employing equations (3.6) and (3.11), we can expand $\Psi_{\lambda}^{(1)}$ in terms of the elements contained in the Virasoro basis of $L_{0}$ eigenstates. For instance, let us expand $\Psi_{\lambda}^{(1)}$ up to level two states

$$
\Psi_{\lambda}^{(1)}=t(\lambda) c_{1}|0\rangle+v(\lambda) c_{-1}|0\rangle+w(\lambda) L_{-2} c_{1}|0\rangle+\cdots
$$

where the coefficients of the expansion $t(\lambda), v(\lambda)$ and $w(\lambda)$ are given by the following integrals

$$
\begin{aligned}
t(\lambda) & =\int_{0}^{\infty} d s d t \frac{e^{-s-t}(\lambda(s+t)+1)^{2} \cos ^{2}\left(\frac{\pi \lambda(s-t)}{2(\lambda(s+t)+1)}\right)}{2 \pi^{2} \lambda \sqrt{s t}}, \\
v(\lambda) & =\int_{0}^{\infty} d s d t \frac{2 e^{-s-t} \sin ^{2}\left(\frac{\pi \lambda(s-t)}{2(\lambda(s+t)+1)}\right)}{\pi^{2} \lambda \sqrt{s t}}, \\
w(\lambda) & =\int_{0}^{\infty} d s d t \frac{e^{-s-t}\left(4-(\lambda(s+t)+1)^{2}\right) \cos ^{2}\left(\frac{\pi \lambda(s-t)}{2(\lambda(s+t)+1)}\right)}{6 \pi^{2} \lambda \sqrt{s t}} .
\end{aligned}
$$

These integrals are convergent provided that the parameter $\lambda$ belongs to the interval $(0,+\infty)$. By performing the change of variables

$$
s \rightarrow \frac{1}{2}(u-u \eta), \quad t \rightarrow \frac{1}{2}(u+u \eta), \quad d s d t \rightarrow \frac{u}{2} d u d \eta,
$$

where $u \in[0, \infty)$ and $\eta \in(-1,1)$, and employing numerical values for the parameter $\lambda$, we are going to numerically evaluate the integrals.

\subsection{Level truncation evaluation of the vacuum energy}

Since in subsection 2.2 we have shown the validity of the equation of motion contracted with the solution itself, we can write the following expression for the normalized value of the vacuum energy

$$
E_{\lambda}=\frac{\pi^{2}}{3}\left\langle\Psi_{\lambda}, Q \Psi_{\lambda}\right\rangle
$$

Plugging the simple tachyon vacuum solution (3.9) into equation (3.17), and using the fact that the second term on the right hand side of equation (3.9) does not contribute since it is a BRST exact term, we obtain

$$
E_{\lambda}=\frac{\pi^{2}}{3}\left\langle\Psi_{\lambda}^{(1)}, Q \Psi_{\lambda}^{(1)}\right\rangle
$$

where the string field $\Psi_{\lambda}^{(1)}$ is defined in equation (3.11). As described in the bosonic case $[1,4,19,24]$, it is convenient to replace the string field $\Psi_{\lambda}^{(1)}$ with $z^{L_{0}} \Psi_{\lambda}^{(1)}$ in the $L_{0}$ level truncation scheme, so that states in the $L_{0}$ level expansion of the solution acquire different integer powers of $z$ at different levels. This parameter $z$ is needed because we will use Padé approximants to evaluate the normalized value of the vacuum energy (3.18). After doing the calculations, we will simply set $z=1$. 
By writing the $c$ ghost in terms of its modes and employing equations (3.6) and (3.11), the string field $\Psi_{\lambda}^{(1)}$ can be readily expanded and the individual coefficients can be numerically integrated. As an example, employing some specific values for the parameter $\lambda$, let us write the string field expanded up to the level fourth states

$$
\begin{aligned}
\Psi_{\lambda=1 / 4}^{(1)}= & 0.94629 c_{1}|0\rangle+0.138297 c_{-1}|0\rangle+0.487297 L_{-2} c_{1}|0\rangle+0.0574864 c_{-3}|0\rangle \\
& -0.19709 L_{-4} c_{1}|0\rangle+0.0390564 L_{-2} c_{-1}|0\rangle+0.166056 L_{-2} L_{-2} c_{1}|0\rangle+\cdots \\
\Psi_{\lambda=1 / 2}^{(1)}= & 0.635128 c_{1}|0\rangle+0.148658 c_{-1}|0\rangle+0.163151 L_{-2} c_{1}|0\rangle+0.085191 c_{-3}|0\rangle \\
& -0.06659 L_{-4} c_{1}|0\rangle+0.0144386 L_{-2} c_{-1}|0\rangle+0.056610 L_{-2} L_{-2} c_{1}|0\rangle+\cdots \\
\Psi_{\lambda=3 / 4}^{(1)}= & 0.543591 c_{1}|0\rangle+0.141941 c_{-1}|0\rangle+0.0544315 L_{-2} c_{1}|0\rangle+0.09104 c_{-3}|0\rangle \\
& -0.02977 L_{-4} c_{1}|0\rangle+0.0006467 L_{-2} c_{-1}|0\rangle+0.031485 L_{-2} L_{-2} c_{1}|0\rangle+\cdots \\
\Psi_{\lambda=1}^{(1)}= & 0.509038 c_{1}|0\rangle+0.13231 c_{-1}|0\rangle-0.00157617 L_{-2} c_{1}|0\rangle+0.08933 c_{-3}|0\rangle \\
& -0.01357 L_{-4} c_{1}|0\rangle-0.0069469 L_{-2} c_{-1}|0\rangle+0.023157 L_{-2} L_{-2} c_{1}|0\rangle+\cdots \\
\Psi_{\lambda=5 / 4}^{(1)}= & 0.498059 c_{1}|0\rangle+0.12281 c_{-1}|0\rangle-0.0371919 L_{-2} c_{1}|0\rangle+0.085046 c_{-3}|0\rangle \\
& -0.00471 L_{-4} c_{1}|0\rangle-0.0112661 L_{-2} c_{-1}|0\rangle+0.020254 L_{-2} L_{-2} c_{1}|0\rangle+\cdots
\end{aligned}
$$

As mentioned previously, to evaluate the normalized value of the vacuum energy (3.18), first we perform the replacement $\Psi_{\lambda}^{(1)} \rightarrow z^{L_{0}} \Psi_{\lambda}^{(1)}$ and then using the resulting string field $z^{L_{0}} \Psi_{\lambda}^{(1)}$, we define

$$
E_{\lambda}(z) \equiv \frac{\pi^{2}}{3}\left\langle z^{L_{0}} \Psi_{\lambda}^{(1)}, Q z^{L_{0}} \Psi_{\lambda}^{(1)}\right\rangle
$$

The normalized value of the vacuum energy (3.18) is obtained just by setting $z=1$ in equation (3.24). As we can see, our problem has been reduced to computation of correlation functions of elements contained in the Virasoro basis of $L_{0}$ eigenstates. Some details regarding this computation are presented in appendix A. Plugging the level expansions (3.19)(3.23) into the definition (3.24), and after evaluating the appropriate correlators, we obtain

$$
\begin{gathered}
E_{\lambda=1 / 4}(z)=-\frac{1.47298}{z^{2}}-0.43054+1.99584 z^{2}+0.21657 z^{4}-2.47629 z^{6}+\cdots, \\
E_{\lambda=1 / 2}(z)=-\frac{0.66354}{z^{2}}-0.31061+0.27892 z^{2}+0.03277 z^{4}-0.34469 z^{6}+\cdots, \\
E_{\lambda=3 / 4}(z)=-\frac{0.48606}{z^{2}}-0.25383+0.01408 z^{2}-0.01013 z^{4}-0.09796 z^{6}+\cdots, \\
E_{\lambda=1}(z)=-\frac{0.42623}{z^{2}}-0.22157-0.05273 z^{2}-0.02121 z^{4}-0.03456 z^{6}+\cdots, \\
E_{\lambda=5 / 4}(z)=-\frac{0.40804}{z^{2}}-0.20123-0.06765 z^{2}-0.02235 z^{4}-0.00978 z^{6}+\cdots
\end{gathered}
$$

As in the bosonic case $[4,20]$, using these kind of series in $z$ for $E_{\lambda}(z)$, we will compute the normalized value of the vacuum energy by the standard procedure based on Padé approximants. To obtain a Padé approximant of order $P_{2+n}^{n}(\lambda, z)$ for the energy, we will need to know the series expansion of $E_{\lambda}(z)$ up to the order $z^{2 n-2}$. 
For the numerical evaluation of the vacuum energy, we have considered the string field $\Psi_{\lambda}^{(1)}$ expanded up to the level twelve states, so that we obtain a series expansion for $E_{\lambda}(z)$ truncated up to the order $z^{22}$. For instance, the explicit expression for the vacuum energy with $\lambda=1 / 4$, which includes contributions from the string field $\Psi_{\lambda=1 / 4}^{(1)}$ truncated up to the level twelve states, is given by

$$
\begin{aligned}
E_{\lambda=1 / 4}(z)= & -\frac{1.472981}{z^{2}}-0.430541+1.995842 z^{2}+0.073704 z^{4}-2.717863 z^{6} \\
& -0.0485597 z^{8}+4.020699 z^{10}+0.098363 z^{12}-6.0537501 z^{14} \\
& +0.033435 z^{16}+9.266269 z^{18}+0.284437 z^{20}-12.515001 z^{22}
\end{aligned}
$$

As an illustration of the numerical method based on Padé approximants, let us compute the normalized value of the vacuum energy using a Padé approximant of order $P_{2+4}^{4}(\lambda, z)$. First, we express $E_{\lambda}(z)$ as the rational function $P_{2+4}^{4}(\lambda, z)$, in this example we consider $\lambda=1 / 4$

$$
E_{\lambda=1 / 4}(z)=P_{2+4}^{4}(1 / 4, z)=\frac{1}{z^{2}}\left[\frac{a_{0}+a_{1} z+a_{2} z^{2}+a_{3} z^{3}+a_{4} z^{4}}{1+b_{1} z+b_{2} z^{2}+b_{3} z^{3}+b_{4} z^{4}}\right] .
$$

Expanding the right hand side of (3.31) around $z=0$ up to sixth order in $z$ and equating the coefficients of $z^{-2}, z^{-1}, z^{0}, z^{1}, z^{2}, z^{3}, z^{4}, z^{5}, z^{6}$ with the expansion (3.30), we get a system of algebraic equations for the unknown coefficients $a_{0}, a_{1}, a_{2}, a_{3}, a_{4}, b_{1}, b_{2}, b_{3}$ and $b_{4}$. Solving those equations we get

$$
\begin{aligned}
& a_{0}=-1.47298, \quad a_{1}=0, \quad a_{2}=-0.805856, \quad a_{3}=0, \quad a_{4}=-0.10585, \\
& b_{1}=0, \quad b_{2}=0.254799, \quad b_{3}=0, \quad b_{4}=1.35235 \text {. }
\end{aligned}
$$

Replacing the value of these coefficients inside the definition of $P_{2+4}^{4}(1 / 4, z)(3.31)$, and evaluating this at $z=1$, we get the following normalized value of the vacuum energy

$$
P_{2+4}^{4}(1 / 4, z=1)=-0.914671336 .
$$

If we naively evaluate the truncated vacuum energy (3.30), i.e., setting $z=1$ in the series before using Padé approximants, we obtain a non-convergent sum. This kind of divergence is also present in the bosonic case [4], where such behavior has appeared in the canonical $L_{0}$ level truncation scheme. And therefore to numerically evaluate the vacuum energy, the use of Padé approximants has been necessary.

The results of our calculations are summarized in table 1. As we can see, the normalized value of the vacuum energy evaluated using Padé approximants confirms the expected analytic result (2.22). Although the convergence to the expected answer gets quite slow, by considering higher level contributions, we will eventually reach to the right value of the vacuum energy $E \rightarrow-1$.

\section{Summary and discussion}

We have analyzed a simple tachyon vacuum solution in cubic superstring field theory. Using this solution, we have tested the validity of the equation of motion contracted with the 


\begin{tabular}{|l|c|c|c|c|c|}
\hline & $P_{2+n}^{n}[\lambda=1 / 4]$ & $P_{2+n}^{n}[\lambda=1 / 2]$ & $P_{2+n}^{n}[\lambda=3 / 4]$ & $P_{2+n}^{n}[\lambda=1]$ & $P_{2+n}^{n}[\lambda=5 / 4]$ \\
\hline$n=0$ & -1.47298157 & -0.66354579 & -0.48606372 & -0.42623492 & -0.40804682 \\
\hline$n=2$ & -1.54937747 & -0.82720585 & -0.72655866 & -0.71701445 & -0.71120414 \\
\hline$n=4$ & -0.91467133 & -0.79944201 & -0.70410295 & -0.74899064 & -0.84724417 \\
\hline$n=6$ & -0.98146315 & -0.90224564 & -0.84739251 & -0.78062826 & -0.87849745 \\
\hline$n=8$ & -0.96055717 & -1.02422931 & -0.89416027 & -0.84178146 & -0.80235684 \\
\hline$n=10$ & -0.98359644 & -0.92679142 & -0.89860136 & -0.84459366 & -0.78508771 \\
\hline$n=12$ & -0.94981557 & -0.93042155 & -1.01894842 & -0.85194204 & -0.78722918 \\
\hline
\end{tabular}

Table 1. The Padé approximation for the normalized value of the vacuum energy $\frac{\pi^{2}}{3}\left\langle z^{L_{0}} \Psi_{\lambda}^{(1)}, Q z^{L_{0}} \Psi_{\lambda}^{(1)}\right\rangle$ evaluated at $z=1$. The results show the $P_{2+n}^{n}$ Padé approximation for various values of the parameter $\lambda$.

solution itself, and have evaluated Ellwood's gauge invariant observable. However, the main result of our paper has been the level truncation analysis of the solution. We have seen that the solution behaves as a regular element in the state space generated by the Virasoro basis of $L_{0}$ eigenstates. We have shown that the computation of the vacuum energy using the level truncated solution brings a value in agreement with Sen's conjecture.

Using the level truncation scheme, it would also be interesting to analyze Schnabl type tachyon vacuum solution which was proposed by Erler [26]. This solution, like the original Schnabl's bosonic solution [1], has an extra term known as the phantom term, this term is necessary for the equation of motion contracted with the solution itself to be satisfied [3]. The level truncation analysis of Schnabl's bosonic solution has been carried out in reference [24], and it has been shown that, to numerically obtain the value of the vacuum energy, the use of Padé approximants was not necessary. It should be nice to see if the same phenomenon can happen in the superstring context, namely if the evaluation of the vacuum energy using the truncated solution leads to a convergent series for the energy.

The main motivation for studying the level truncation analysis of tachyon vacuum solutions in cubic superstring field theory is to prepare a numerical background in order to analyze more cumbersome solutions, such as the multibrane solutions [34, 35], and the recently proposed Erler's analytic solution for tachyon condensation in Berkovits non-polynomial open superstring field theory [37]. The presence of higher interaction terms in Berkovits string field theory action, will bring us challenges for the level truncation analysis of Erler's solution. In a future work, we would like to extend the results of our paper, for instance, to evaluate higher interaction vertices, we will look for an alternative calculation method that avoids the computation of finite conformal transformations. These methods known as conservation laws [21, 46, 47] already exist, and we will need to implement them for our purposes.

\section{Acknowledgments}

I would like to thank Ted Erler, Isao Kishimoto, and Michael Kroyter for useful discussions. This work is supported by CNPq grant 303073/2012-8. 


\section{A Details on the level truncation evaluation of the vacuum energy}

We first consider some of the ingredients for the calculation of the vacuum energy, then show a particular example in detail. First of all, recall that for the cubic superstring field theory, the inner product appearing in the evaluation of the vaccum energy is the standard BPZ inner product with the difference that we must insert the operator $Y_{-2}$ at the open string midpoint. The operator $Y_{-2}$ can be written as the product of two inverse picture changing operators $Y_{-2}=Y(i) Y(-i)$, where $Y(z)=-\partial \xi e^{-2 \phi} c(z)$.

A general inner product of two vertex operators $\langle A, B\rangle$ is given by the evaluation of the following correlator

$$
\langle A, B\rangle=\left\langle Y_{-2}(\mathcal{I} \circ A(0)) B(0)\right\rangle,
$$

where $\mathcal{I}(z)=-1 / z$, is the usual conformal transformation that defines the BPZ inner product. This definition, together with the identity

$$
\begin{aligned}
& \left\langle\prod_{i=1}^{n} \xi\left(x_{i}\right) \prod_{j=1}^{n} \eta\left(y_{j}\right) \prod_{k=1}^{m} b\left(u_{k}\right) \prod_{l=1}^{m+3} c\left(v_{l}\right) \prod_{s=1}^{p} e^{q_{s} \phi\left(z_{s}\right)}\right\rangle \\
& =-\prod_{i<i^{\prime}}\left(x_{i}-x_{i^{\prime}}\right) \prod_{j<j^{\prime}}\left(y_{j}-y_{j^{\prime}}\right) \prod_{i, j}\left(x_{i}-y_{j}\right)^{-1} \prod_{k<k^{\prime}}\left(u_{k}-u_{k^{\prime}}\right) \prod_{l<l^{\prime}}\left(v_{l}-v_{l^{\prime}}\right) \prod_{k, l}\left(u_{k}-v_{l}\right)^{-1} \\
& \quad \times \prod_{s<s^{\prime}}\left(z_{s}-z_{s^{\prime}}\right)^{-q_{s} q_{s^{\prime}}}
\end{aligned}
$$

and the expression for the bosonized representation of the superconformal ghost $\gamma=\eta e^{\phi}$, allows us to compute the relevant terms which appear in the level truncation analysis of the vacuum energy.

For instance, employing the above identity (A.2), we have obtained some correlation functions that are very useful in the evaluation of the vacuum energy presented in subsection 3.2 , let us list these correlators

$$
\begin{aligned}
& \left\langle Y_{-2} c(x) \gamma(y) \gamma(z)\right\rangle=\frac{1}{2}\left(1+x^{2}\right)(1+y z) \\
& \left\langle Y_{-2} T(w) c(x) \gamma(y) \gamma(z)\right\rangle \\
& =\frac{x^{2}-2 w x-1}{\left(1+w^{2}\right)(w-x)^{2}}\left\langle Y_{-2} c(x) \gamma(y) \gamma(z)\right\rangle+\frac{1+x^{2}}{\left(1+w^{2}\right)(w-x)}\left\langle Y_{-2} \partial_{x} c(x) \gamma(y) \gamma(z)\right\rangle \\
& \quad+\frac{y^{2}-2 w y-1}{2\left(1+w^{2}\right)(w-y)^{2}}\left\langle Y_{-2} c(x) \gamma(y) \gamma(z)\right\rangle+\frac{1+y^{2}}{\left(1+w^{2}\right)(w-y)}\left\langle Y_{-2} c(x) \partial_{y} \gamma(y) \gamma(z)\right\rangle \\
& \quad+\frac{z^{2}-2 w z-1}{2\left(1+w^{2}\right)(w-z)^{2}}\left\langle Y_{-2} c(x) \gamma(y) \gamma(z)\right\rangle+\frac{1+z^{2}}{\left(1+w^{2}\right)(w-z)}\left\langle Y_{-2} c(x) \gamma(y) \partial_{z} \gamma(z)\right\rangle .
\end{aligned}
$$

In general a correlation function of the form $\left\langle T(w) \Phi_{1}(x) \Phi_{2}(y) \cdots\right\rangle$, where $\Phi_{i}$ is a primary field with conformal weight $h_{i}$, can be computed using the following expression

$$
\begin{aligned}
& \left\langle Y_{-2} T(w) \Phi_{1}(x) \Phi_{2}(y) \cdots\right\rangle \\
& =\frac{h_{1}\left(1+2 w x-x^{2}\right)}{\left(1+w^{2}\right)(w-x)^{2}}\left\langle Y_{-2} \Phi_{1}(x) \Phi_{2}(y) \cdots\right\rangle+\frac{1+x^{2}}{\left(1+w^{2}\right)(w-x)}\left\langle Y_{-2} \partial_{x} \Phi_{1}(x) \Phi_{2}(y) \cdots\right\rangle
\end{aligned}
$$




$$
\begin{aligned}
& +\frac{h_{2}\left(1+2 w y-y^{2}\right)}{\left(1+w^{2}\right)(w-y)^{2}}\left\langle Y_{-2} \Phi_{1}(x) \Phi_{2}(y) \cdots\right\rangle+\frac{1+y^{2}}{\left(1+w^{2}\right)(w-y)}\left\langle Y_{-2} \Phi_{1}(x) \partial_{y} \Phi_{2}(y) \cdots\right\rangle \\
& +\cdots
\end{aligned}
$$

As a pedagogical illustration, we are going to show some steps in the computation of the normalized value of the vacuum energy for a string field expanded up to level two states. The expression for this string field is given in equation (3.12), and it can be written using the corresponding vertex operators as follows

$$
\Psi_{\lambda}^{(1)}=t(\lambda) c+\frac{v(\lambda)}{2} \partial^{2} c+w(\lambda) T c
$$

Plugging the string field (A.6) into the definition of the normalized value of the vacuum energy (3.24), we obtain

$$
\begin{aligned}
E_{\lambda}(z)=\frac{\pi^{2}}{3}[ & \frac{t^{2}(\lambda)}{z^{2}}\langle c, Q c\rangle+\frac{v^{2}(\lambda) z^{2}}{4}\left\langle\partial^{2} c, Q \partial^{2} c\right\rangle+w^{2}(\lambda) z^{2}\langle T c, Q T c\rangle \\
& \left.+t(\lambda) v(\lambda)\left\langle\partial^{2} c, Q c\right\rangle+2 t(\lambda) w(\lambda)\langle T c, Q c\rangle+v(\lambda) w(\lambda) z^{2}\left\langle T c, Q \partial^{2} c\right\rangle\right] .
\end{aligned}
$$

All correlation functions appearing in equation (A.7) can be evaluated using the correlators (A.3)-(A.5). For instance, let us explicitly compute the correlator $\langle T c, Q c\rangle$, since $Q c=c \partial c-\gamma^{2}$, the non-vanishing contribution in the correlator $\langle T c, Q c\rangle$ is given by

$$
\langle T c, Q c\rangle=-\left\langle T c, \gamma^{2}\right\rangle
$$

Employing the definition of the BPZ inner product (A.1), we can evaluate the above correlator by using a parameter $\epsilon$ eventually taken to zero

$$
\langle T c, Q c\rangle=-\lim _{\epsilon \rightarrow 0}\left\langle Y_{-2} \mathcal{I} \circ(T(\epsilon) c(\epsilon)) \gamma^{2}(\epsilon)\right\rangle
$$

performing the conformal transformation of the vertex operator $T c$ and using equation (A.4), we obtain

$$
\lim _{\epsilon \rightarrow 0}\left\langle Y_{-2} \mathcal{I} \circ(T(\epsilon) c(\epsilon)) \gamma^{2}(\epsilon)\right\rangle=0 .
$$

By the same procedure, we can compute all the remaining correlators appearing in equation (A.7). Therefore at the end we arrive to an expression for the vacuum energy corresponding to a string field truncated up to level two states

$$
E_{\lambda}(z)=\frac{\pi^{2}}{3}\left[-\frac{t^{2}(\lambda)}{2 z^{2}}-\frac{v^{2}(\lambda) z^{2}}{2}+2 w^{2}(\lambda) z^{2}-t(\lambda) v(\lambda)+v(\lambda) w(\lambda) z^{2}\right] .
$$

Open Access. This article is distributed under the terms of the Creative Commons Attribution License (CC-BY 4.0), which permits any use, distribution and reproduction in any medium, provided the original author(s) and source are credited. 


\section{References}

[1] M. Schnabl, Analytic solution for tachyon condensation in open string field theory, Adv. Theor. Math. Phys. 10 (2006) 433 [hep-th/0511286] [InSPIRE].

[2] E. Witten, Noncommutative geometry and string field theory, Nucl. Phys. B 268 (1986) 253 [INSPIRE].

[3] Y. Okawa, Comments on Schnabl's analytic solution for tachyon condensation in Witten's open string field theory, JHEP 04 (2006) 055 [hep-th/0603159] [INSPIRE].

[4] T. Erler and M. Schnabl, A simple analytic solution for tachyon condensation, JHEP 10 (2009) 066 [arXiv:0906.0979] [INSPIRE].

[5] M. Schnabl, Algebraic solutions in open string field theory - a lightning review, Acta Polytechnica 50 (2010) 102 [arXiv: 1004.4858] [INSPIRE].

[6] Y. Okawa, Analytic methods in open string field theory, Prog. Theor. Phys. 128 (2012) 1001 [INSPIRE].

[7] M. Kiermaier, Y. Okawa and P. Soler, Solutions from boundary condition changing operators in open string field theory, JHEP 03 (2011) 122 [arXiv:1009.6185] [INSPIRE].

[8] M. Kiermaier, Y. Okawa, L. Rastelli and B. Zwiebach, Analytic solutions for marginal deformations in open string field theory, JHEP 01 (2008) 028 [hep-th/0701249] [INSPIRE].

[9] L. Bonora and S. Giaccari, Generalized states in SFT, Eur. Phys. J. C 73 (2013) 2644 [arXiv: 1304.2159] [INSPIRE].

[10] L. Bonora, S. Giaccari and D.D. Tolla, Analytic solutions for Dp-branes in SFT, JHEP 12 (2011) 033 [arXiv:1106.3914] [INSPIRE].

[11] S. Zeze, Application of KBc subalgebra in string field theory, Prog. Theor. Phys. Suppl. 188 (2011) 56 [InSPIRE].

[12] M. Murata and M. Schnabl, Multibrane solutions in open string field theory, JHEP 07 (2012) 063 [arXiv:1112.0591] [INSPIRE].

[13] H. Hata and T. Kojita, Singularities in K-space and multi-brane solutions in cubic string field theory, JHEP 02 (2013) 065 [arXiv: 1209.4406] [INSPIRE].

[14] T. Erler and C. Maccaferri, String field theory solution for any open string background, JHEP 10 (2014) 029 [arXiv:1406.3021] [INSPIRE].

[15] H. Hata and T. Kojita, Inversion symmetry of gravitational coupling in cubic string field theory, JHEP 12 (2013) 019 [arXiv:1307.6636] [INSPIRE].

[16] T. Erler, Split string formalism and the closed string vacuum, JHEP 05 (2007) 083 [hep-th/0611200] [INSPIRE].

[17] I. Ellwood, The closed string tadpole in open string field theory, JHEP 08 (2008) 063 [arXiv:0804.1131] [INSPIRE].

[18] M. Schnabl, Wedge states in string field theory, JHEP 01 (2003) 004 [hep-th/0201095] [INSPIRE].

[19] E.A. Arroyo, Cubic interaction term for Schnabl's solution using Padé approximants, J. Phys. A 42 (2009) 375402 [arXiv:0905.2014] [INSPIRE].

[20] E. Aldo Arroyo, Level truncation analysis of regularized identity based solutions, JHEP 11 (2011) 079 [arXiv:1109.5354] [INSPIRE]. 
[21] E.A. Arroyo, Conservation laws and tachyon potentials in the sliver frame, JHEP 06 (2011) 033 [arXiv: 1103.4830] [INSPIRE].

[22] E.A. Arroyo, Comments on regularization of identity based solutions in string field theory, JHEP 11 (2010) 135 [arXiv:1009.0198] [INSPIRE].

[23] E. Aldo Arroyo, The tachyon potential in the sliver frame, JHEP 10 (2009) 056 [arXiv: 0907.4939] [INSPIRE].

[24] T. Takahashi, Level truncation analysis of exact solutions in open string field theory, JHEP 01 (2008) 001 [arXiv:0710.5358] [INSPIRE].

[25] I.Y. Arefeva, P.B. Medvedev and A.P. Zubarev, New representation for string field solves the consistency problem for open superstring field theory, Nucl. Phys. B 341 (1990) 464 [INSPIRE].

[26] T. Erler, Tachyon vacuum in cubic superstring field theory, JHEP 01 (2008) 013 [arXiv: 0707.4591] [INSPIRE].

[27] E.A. Arroyo, Generating Erler-Schnabl-type solution for tachyon vacuum in cubic superstring field theory, J. Phys. A 43 (2010) 445403 [arXiv: 1004.3030] [INSPIRE].

[28] T. Erler, Exotic universal solutions in cubic superstring field theory, JHEP 04 (2011) 107 [arXiv: 1009.1865] [INSPIRE].

[29] I.Y. Aref'eva, R.V. Gorbachev and P.B. Medvedev, Tachyon solution in cubic Neveu-Schwarz string field theory, Theor. Math. Phys. 158 (2009) 320 [arXiv:0804.2017] [INSPIRE].

[30] R.V. Gorbachev, New solution of the superstring equation of motion, Theor. Math. Phys. 162 (2010) 90 [Teor. Mat. Fiz. 162 (2010) 106] [InSPIRE].

[31] I.Y. Aref'eva, R.V. Gorbachev and P.B. Medvedev, Pure gauge configurations and solutions to fermionic superstring field theories equations of motion, J. Phys. A 42 (2009) 304001 [arXiv: 0903.1273] [INSPIRE].

[32] I.Y. Arefeva and R.V. Gorbachev, On gauge equivalence of tachyon solutions in cubic Neveu-Schwarz string field theory, Theor. Math. Phys. 165 (2010) 1512 [arXiv:1004.5064] [INSPIRE].

[33] M. Kroyter, Comments on superstring field theory and its vacuum solution, JHEP 08 (2009) 048 [arXiv:0905.3501] [INSPIRE].

[34] E. Aldo Arroyo, Comments on multibrane solutions in cubic superstring field theory, Prog. Theor. Exp. Phys. 2014 (2014) 063B03 [arXiv:1306.1865] [InSPIRE].

[35] E. Aldo Arroyo, Multibrane solutions in cubic superstring field theory, JHEP 06 (2012) 157 [arXiv: 1204.0213] [INSPIRE].

[36] N. Berkovits, SuperPoincaré invariant superstring field theory, Nucl. Phys. B 450 (1995) 90 [Erratum ibid. B 459 (1996) 439] [hep-th/9503099] [INSPIRE].

[37] T. Erler, Analytic solution for tachyon condensation in Berkovits' open superstring field theory, JHEP 11 (2013) 007 [arXiv:1308.4400] [INSPIRE].

[38] V.A. Kostelecky and S. Samuel, The static tachyon potential in the open bosonic string theory, Phys. Lett. B 207 (1988) 169 [INSPIRE].

[39] V.A. Kostelecky and S. Samuel, On a nonperturbative vacuum for the open bosonic string, Nucl. Phys. B 336 (1990) 263 [inSPIRE]. 
[40] N. Moeller and W. Taylor, Level truncation and the tachyon in open bosonic string field theory, Nucl. Phys. B 583 (2000) 105 [hep-th/0002237] [INSPIRE].

[41] W. Taylor, A perturbative analysis of tachyon condensation, JHEP 03 (2003) 029 [hep-th/0208149] [INSPIRE].

[42] D. Gaiotto and L. Rastelli, Experimental string field theory, JHEP 08 (2003) 048 [hep-th/0211012] [INSPIRE].

[43] K. Ohmori, Level expansion analysis in NS superstring field theory revisited, hep-th/0305103 [INSPIRE].

[44] J. Raeymaekers, Tachyon condensation in string field theory: tachyon potential in the conformal field theory approach, Ph.D. thesis, KULeuven, Leuven Belgium (2001).

[45] P.-J. De Smet and J. Raeymaekers, Level four approximation to the tachyon potential in superstring field theory, JHEP 05 (2000) 051 [hep-th/0003220] [INSPIRE].

[46] P.-J. De Smet, Tachyon condensation: calculations in string field theory, hep-th/0109182 [INSPIRE].

[47] L. Rastelli and B. Zwiebach, Tachyon potentials, star products and universality, JHEP 09 (2001) 038 [hep-th/0006240] [INSPIRE]. 